\title{
Comparisons between CCD and CMOS based on Freescale
}

\author{
Zhi-Wei WANG, Xiang SHI \\ Mechanical Engineering and Automation, Shanghai Ocean University, Shanghai, China
}

\begin{abstract}
With the rise of digital camera, mobile phone camera, image sensor is becoming one of the most dazzling star around the semiconductor products, and in the image sensor, CCD sensor which is exclusive by Japanese and the CMOS sensor that be contention of a hundred schools of thought are trying to overcome its own shortcomings, both of them hope to become a mainstream technology on the market. So the article mainly introduces CCD (Charge coupled device) and CMOS (Complementary Metal Oxide Semiconductor) camera type in the application of the Freescale, and also including the advantages and the disadvantages of the two cameras in the actual competition. And provide a reference for the choice of camera group in the Freescale Cup!
\end{abstract}

KEYWORD: Freescale; CCD; CMOS; Smartca

\section{INSTRUCTION}

The national college students The Freescale Cup comprises a photoelectric group, camera group and electromagnetic group, where in the camera group the smart car is the fastest, and attracts much attention. In the selected camera types mainly include CMOS and CCD. In the competition, when the car into the starting state. CCD camera to collect real-time traffic information, and then the microcontroller will identify the road and realize the tracking control of the information. Using the time to control DC motor speed and position PID control steering gear optimization, the dynamic adjustment of PID parameters in the black line of location based. These measures ensure travel along a specified route safety and fast. So as to realize the automatic tracking. The first step is to the analog signal output by the camera A/D conversion A/D collection to DSP, then the original image data processing the collected. In order to collect image information. In the camera's choice, should consider the resolution, field size, image clarity, color contrast, power, mass and volume. While CCD and COMS in these areas has its own merits.

\section{THE WHOLE SCHEME DESIGN}

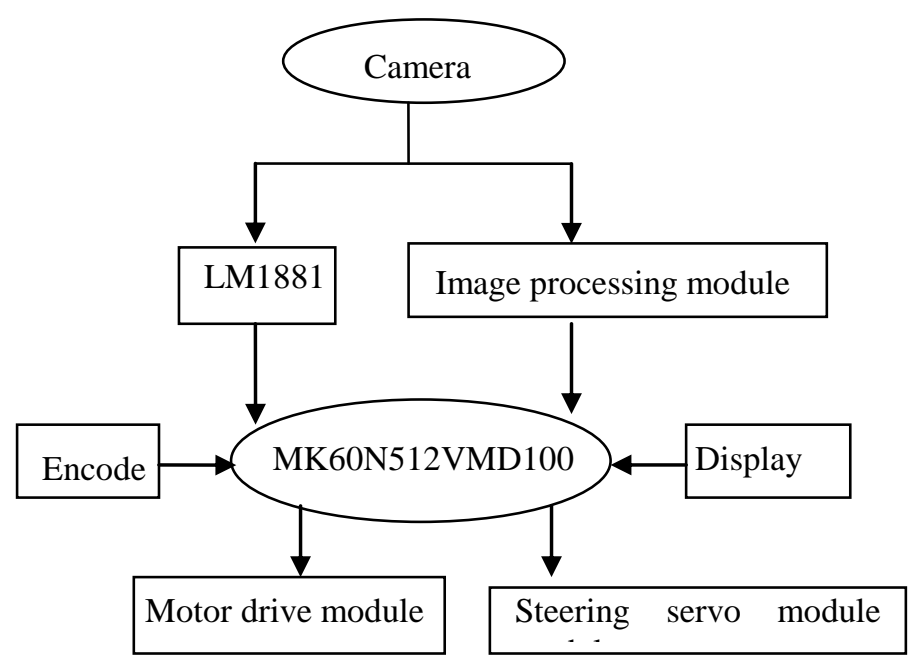

Fig. 1 : vehicle overall structure diagram

Intelligent vehicle overall working mode: image sensor acquisition circuit information, and output the PAL signal, through the image processing module into image binaryzation. Then using LM1881 for video synchronous separator, binarizating the image signal, pirating field signal. Afterwards input all these signal into microcontroller MK60N512VMD100. Through the two-dimensional array access information of the track; Through the encoder to detect speed racing driving, And the use of mk60 input pulse calculation to obtain the speed and distance of capture function, steering engine by 
PD control, through the PMW control to adjust the speed of the motor driving circuit.

\section{CCD MODULE CIRCUIT DESIGN}

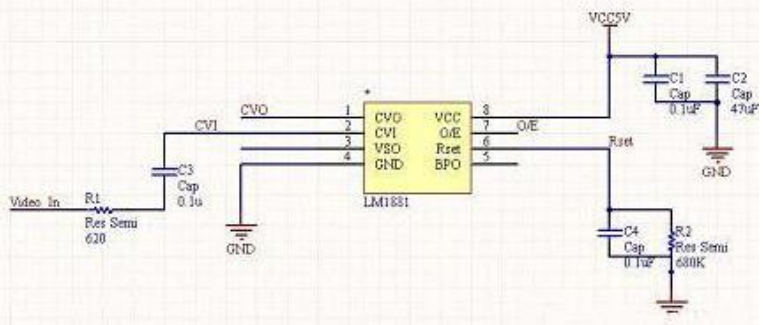

Fig. 2: Video signal separation circuit

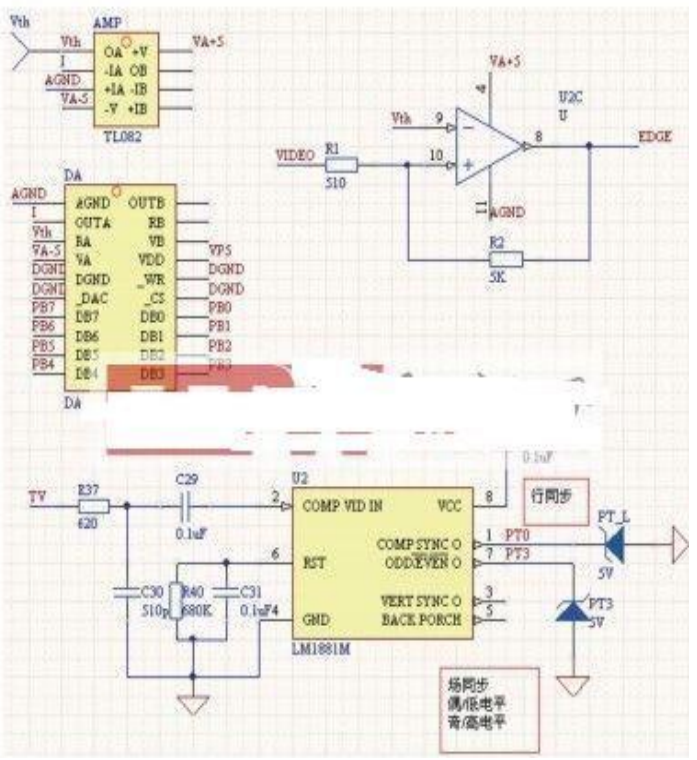

Fig. 3: CCD Module Circuit

Using LM1881 video separation chip can imitate video signal separating into parity synchronization signal and line synchronizing signal.

A. Parity synchronization signal. The output of the synchronous parity signal is high/low lever, odd field output high lever, even field output low lever, which can be directly connected with the Single chip microcomputer common I/O port. The output image of the camera whether odd or even just through judging the $\mathrm{I} / \mathrm{O}$ port is high or low.

B.Line synchronizing signal. Line synchronizing signal into a series of pulse signal, which is directly connected to the external single chip microcontroller port in IRQ, and the terminal is set as falling edge trigger. Each row synchronization signal arrival would induce a external interrupt. We can count on the interrupt, then we can know the current interruption for which a line.

\section{CMOS MODULE CIRCUIT DESIGN}

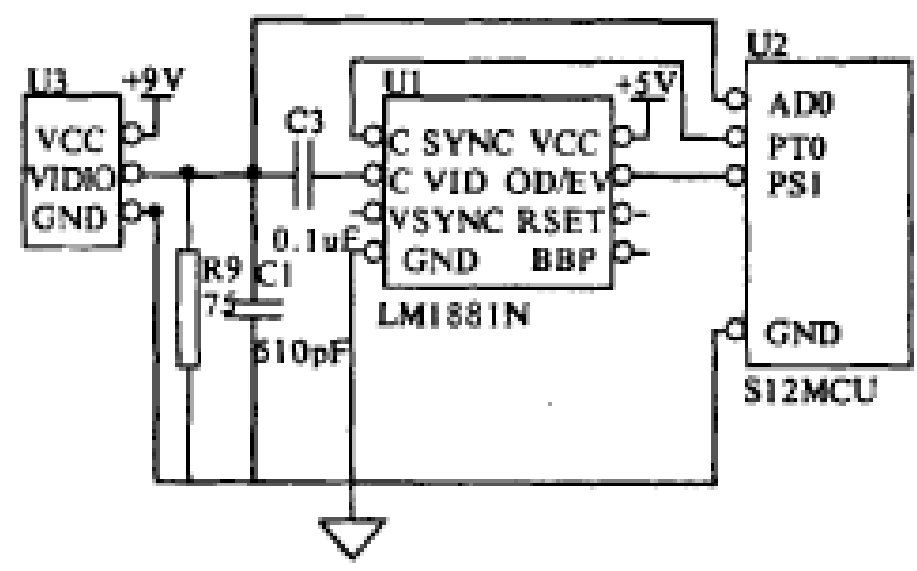

Fig. 4: Sampling Circuit

Car video sampling circuit as it is shown in Figure 4.it is composed of a camera, a LM1881 circuit and a S12 controller of wisdom.

The video principles are summarized as follows. When the PS1 port flip, show a new image to come, Image sampling start. When the PS0 detect a rising edge synchronous signal, it means a new image data is outputting. The $\mathrm{S} 12$ controller sampling on the AD0 port video signal until the next line synchronizing signal to the PT0 port so far, it presents that this row of image sampling is completed. And then repeat the above steps.

\section{COMPARISON OF CCD AND COMS}

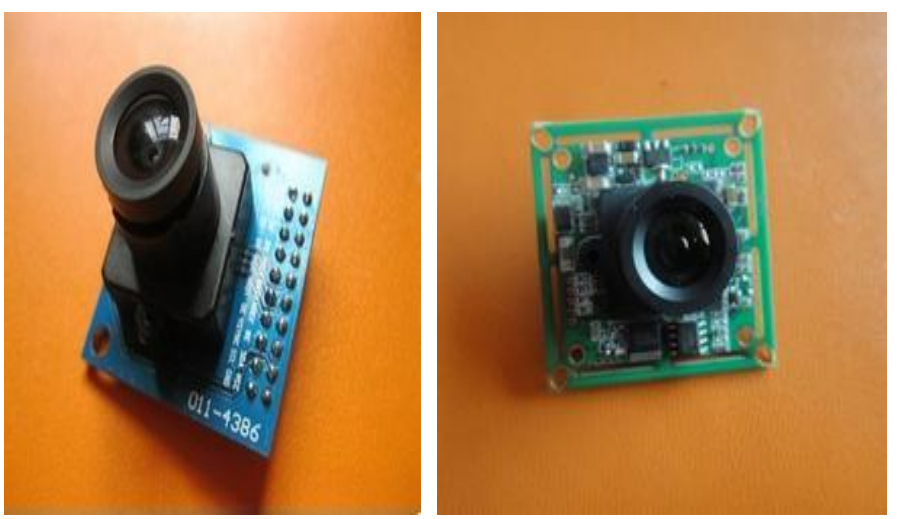

Fig. 5: CCD and CMOS

CCD and CMOS circuit and its principles are introduced through the above. So we can make a detailed comparison from a technical point of view. Specific differences are as shown below. 
Table1:Parameter Comparison

\begin{tabular}{|l|l|l|}
\hline & CCD & CMOS \\
\hline Sensitivity & The same area of high & Photosensitive opening is small, low sensitivity \\
\hline Cost & High cost & Low cost \\
\hline Resolution & Connection complexity, high resolution & Low \\
\hline Noise & A single amplifier, low noise & High amplification, high noise \\
\hline Consumption & Additional voltage, high consumption & Direct amplification, low consumption \\
\hline
\end{tabular}

ISO sensitivity difference: Because each pixel contains a CMOS amplifier and a A/D circuit, additional equipment compressed the surface area of the single pixel photosensitive. Therefore, the same size photoreceptor dimensions under the same pixel CCD have higher sensitivity than CMOS.

- Resolution: On the first point sensitivity differences, because the structure of CMOS for each pixel is more complex than CCD, The photosensitive opening less than $\mathrm{CCD}, \mathrm{CCD}$ sensor resolution is usually better than CMOS when CCD and CMOS are relatively the same photoreceptor size.

- Cost: CMOS is commonly used in the MOS programming in the semiconductor industry, it can integrate all of the surrounding facilities in a single chip, which save the processing chip needed to afford the cost and yield loss, CCD output information with charge transfer mode comparing to the CMOS, which must open another transmission channel. if there is a fault in the pixel channel, it will lead to a whole row of signal disruption. So the rate of finish products of CCD is lower than CMOS, besides due to the additional delivery channel and other channels surrounding ADC, The manufacturing cost of $\mathrm{CCD}$ is higher than that of CMOS.

- Consumption: CMOS image charge drive mode is active, charge produced by photosensitive diode is directly output magnified by the next transistor. But the CCD work mode is passive, which must be applied additional voltage to let each pixel movement of charge to transmission channel. The additional voltage usually requires more than $12 \mathrm{~V}$. However, CMOS image sensor power generally small, only $5 \mathrm{~V}$ can work normally, or even $3.3 \mathrm{~V}$ models. Compared with the input requirements of TTL at $12 \mathrm{~V}$, power supply system of CMOS sensor are compatible with the majority of the chip and the control circuit. The circuit greatly simplified without the extra boost circuit, which improved reliability.
Noise: The CCD unique feature can maintain signal fully during transmission without distortion. Through each pixel send to a single amplifier and then handle together, so image can keep a high integrity; the production of CMOS is simple because there is no special design channel. So each pixel must be magnified before all these integrated. Considering all these factors, the noise is more susceptible to CMOS imaging process.

\section{CONCLUSIONS}

From the technical level of the CCD sensor in sensitivity, resolution, noise control is better than that of CMOS sensor, while the CMOS sensor has the advantages of low cost, low power consumption, and highly integrated features. However, in the actual competition the venue of light intensity, models of its own weight and power requirements for battery life would also affect the camera selection. We need considerate the comprehensive performance of $\mathrm{CCD}$ and $\mathrm{CMOS}$ in the above factors!

\section{REFERENCES}

[1] J. X. Sui, L. Yang, Y. Zhang: The Freescale Cup (Publishing House of Electronics Industry, China)

[2] K. Yun, C. Gao: Journal of North China Institute of Water Conservancy and Hydroelectric Power, Vol.29 (2008) No. $5, \mathrm{p} 56$.

[3] Z. C. Song, X. M. Li: 2010 International Conference on Computer and Communication Technologies in Agriculture Engineering (June12-13, 2010), Vol10, p102.

[4] National Semiconductor," LM1881 video syc separator," Data Sheet: DS009150, Apr.2001.

[5] Techwell, Inc, 'Enhanced NTSC/Pal/SECAM Video Decoder," Data Sheet: TW99, Mar. 30, 2000.

[6] Q. Zhuo, J. wang, L, Wang: Detection method of circuit parameters based on CCD, Electronic Engineering \& Product World, 2006,18 (7): P143-145.

[7] K. S. Huang, H. M. Jin, D. N. Jiang: South Korea Intelligent Model Car Technology Scheme Analysis, Electronic Engineering \& Product World,2006,14 (5): P150-152.

[8] http://ee.ofweek.com/2013-11/ART-11000-280628748770.html 